\title{
Chronic epipharyngitis: A missing trigger in chronic fatigue syndrome
}

\author{
Osamu Hotta ${ }^{1,2 *}$, Norio Ieiri ${ }^{1}$, Chiyoko N Inoue ${ }^{3}$ and Ayaki Tanaka ${ }^{4}$ \\ ${ }^{1}$ Division of Internal Medicine, Hotta Osamu Clinic, Sendai, Japan \\ ${ }^{2}$ Division of Nephrology and Endocrinology, Tohoku Medical and Pharmaceutical University, Sendai, Japan \\ ${ }^{3}$ Department of Pediatrics, Red Cross Sendai Hospital, Sendai, Japan \\ ${ }^{4}$ Tanaka ENT Clinic, Osaka, Japan
}

\begin{abstract}
Located at the back of the nasal cavities, epipharynx is an immunologically activated site even under normal conditions, and enhanced immunological activation is prone to occur in response to infectious pathogens, air dusts, chemicals, and other stimulating substances. Chronic epipharyngitis is not a widely understood condition. However, because of its close link with the nervous system and both the innate and acquired immune systems, it may play an important role as a trigger for the development of neuroendocrine disorders, including chronic fatigue syndrome and other somatic symptoms. Thus, the "epipharynx-brain interaction" is worth consideration in managing patients with chronic fatigue syndrome. Given that chronic epipharyngitis and its brain interactions are not fully understood, it is important to focus future research on this condition.
\end{abstract}

Chronic fatigue syndrome (CFS)-also called myalgic encephalomyelitis/chronic fatigue syndrome (ME/CFS) - is defined exclusively by clinical symptoms; however, recent studies indicate that the symptoms of CFS reflect low-grade inflammation in the brain $[1,2]$. If this is the case, it is important to determine what might trigger that inflammation.

In some cases, the activation of the immune system in the brain can be caused by a trigger inside the brain, such as an infectious agent or autoimmune process. In other cases, the activation of the immune system in the brain may be caused by activation of innate immunity outside the brain [2]. Activation of innate immunity outside the brain produces neuroexcitatory molecules, such as pro-inflammatory cytokines, nitrous oxide, reactive oxygen species, and prostaglandins. Glial cells that envelop both the vagus nerve and sensory vagus paraganglia have chemoreceptors for these molecules [3]; notably, activation of the chemoreceptors generates retrograde signals that travel up the vagus nerve to the nucleus tractus solitarius (NTS), which then activates the innate immune system of the brain [4].

Adjuvants increase the innate immune response by augmenting the activities of dendritic cells (DCs), lymphocytes, and macrophages. Moreover, it has been proposed that adjuvants may cause CFS-like symptoms, designated as autoimmune/inflammatory syndrome induced by adjuvants (ASIA) [5]. The concept of ASIA includes siliconosis, macrophagic myofasciitis, Gulf war syndrome, postvaccination phenomena, and sick building syndrome, which have all been linked with previous exposure to an adjuvant. Clinical symptoms in ASIA include myalgia, arthralgia, chronic fatigues, neurological cognitive impairment, fever, gastrointestinal symptoms, respiratory symptoms, skin manifestations, and the appearance of autoantibodies. Most of these symptoms of ASIA coincide with those of ME/CFS.

Located at the back of the nasal cavities, the epipharynx is a unique tissue that is vulnerable to the effects of upper respiratory infections and air pollution. Concurrently, as a component of the nasopharynxassociated lymphoid tissue, the epipharynx plays a role in both innate and acquired immunity through its abundance of DCs and adaptive immune cells [6].

Between October 2014 and January 2018, in 93 young women who exhibited serious somatic symptoms following human papillomavirus (HPV) vaccination, we examined the epipharynx using a previously described method $[7,8]$. Surprisingly, all patients demonstrated severe chronic epipharyngitis; further, 42 of them (45.2\%) reported pharyngeal symptoms, such as throat pain and throat discomfort. Notably, throat pain is a classical symptom frequently observed in patients with CFS. Interestingly, the inflammation of the epipharynx is not felt in the upper pharynx in the back of the nose, but presents as a pain in the middle or lower pharynx; epipharyngitis is the most frequent cause of throat pain [9]. A characteristic of chronic epipharyngitis is easy bleeding, due to congestion [6-8]. Worthy of particular mention is that chronic epipharyngitis is difficult to diagnose by standard endoscopic procedures, as the surface of the epipharyngeal wall is optically unremarkable in patients with chronic epipharyngitis. The diagnosis of chronic epipharyngitis can be achieved by epipharyngeal abrasive procedures, using a cotton swab with optimized bleeding during abrasion [6-8]. Thus, it is highly likely that chronic epipharyngitis has been overlooked through routine examination by physicians.

Correspondence to: Osamu Hotta, Division of Internal Medicine, Hotta Osamu Clinic; Sendai, Miyagi 984-0013, Japan, Tel. 81-22-390-6033, Fax: 81-22-3906035, E-mail: hottao@remus.dti.ne.jp

Key words: chronic epipharyngitis, chronic fatigue syndrome, epipharyngeal abrasive therapy, epipharynx-brain interaction

Received: March 03, 2018; Accepted: March 19, 2018; Published: March 21, 2018 


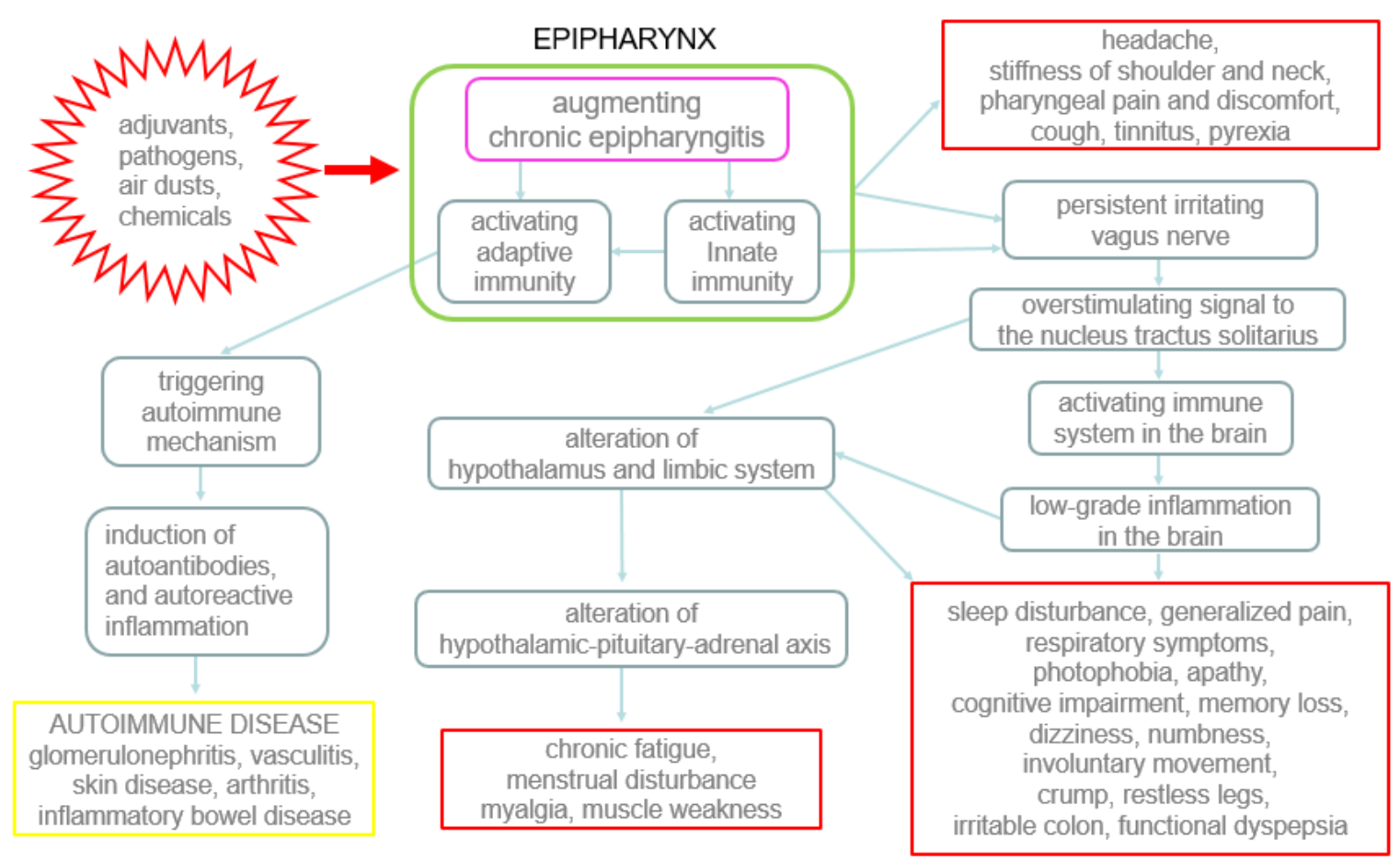

Figure 1. Possible relationship of chronic epipharyngitis with chronic fatigue syndrome and autoimmune diseases.

Vaccine adjuvants, infectious pathogens, air dusts, and other stimulating substances augment chronic epipharyngitis. This condition activates epipharyngeal inner immunity and produces neuroexcitatory molecules. These molecules generate retrograde signals that travel up the vagus nerve to the nucleus tractus solitarius, which then activates the immune system of the brain, resulting in low-grade inflammation in the brain. As a result of the epipharynx-brain interaction, together with the symptoms of epipharyngitis itself, various clinical symptoms of chronic fatigue syndrome develop (red frame). Moreover, activated epipharyngeal acquired immunity may trigger autoimmune mechanisms, resulting in the development of autoimmune diseases (yellow frame).

The epipharynx receives autonomic input via the pharyngeal plexus, which consists of afferent and efferent fibers from the glossopharyngeal nerve and vagus nerve, as well as sympathetic efferent fibers from the cervical ganglia [10]. Vagus nerve endings in the epipharynx could be irritated by noxious substances present in chronic epipharyngitis, including pro-inflammatory cytokines, reactive oxygen species, inflammatory mediators, cell debris, and pathogenic antigens, all of which can stimulate or damage the unmyelinated vagus nerve endings, triggering persistently disorganized and abnormal nerve signals.

Thus, it is conceivable that the exposure of the epipharyngeal surface to adjuvants, infectious pathogens, chemicals, and other stimulating substances, which exacerbate the inflammation of chronic epipharyngitis, may activate epipharyngeal innate immunity by producing neuroexcitatory molecules. These molecules generate retrograde signals that travel up the vagus nerve to the NTS, which then activates the immune system of the brain, resulting in the lowgrade inflammation in the brain that develops into CFS. Additionally, activated epipharyngeal innate immunity enhances the adaptive immune system, which may initiate an autoimmune reaction, including autoantibody production (Figure 1).

In support of this concept, a significant improvement in symptoms, following epipharyngeal abrasive therapy with a $\mathrm{ZnCl} 2$ solution was obtained in patients with CFS following HPV vaccination [6].

In conclusion, chronic epipharyngitis is not a well-known condition. However, it may play an important role as a trigger in the development of neuroendocrine disorders, including ME/CFS and ASIA, via the socalled "epipharynx-brain interaction."
Additionally, we suggest that the condition of epipharynx may be worth examining in the broader context of the treatment of autoimmune diseases and neuroendocrine disorders, regardless of the initial cause. Given that chronic epipharyngitis and its brain interactions are not fully understood, we believe that it is important to focus future research on this condition.

\section{Conflicts of interest}

The authors declare that they have no conflicts of interest.

\section{Disclosure}

This research did not receive any specific grant from funding agencies in the public, commercial, or not-for-profit sectors.

\section{References}

1. Montoya JG, Holmes TH, Anderson JN, Maecker HT, Rosenberg-Hasson Y, et al. (2017) Cytokine signature associated with disease severity in chronic fatigue syndrome patients. Proc Natl Acad Sci U S A 114(34): E7150-E7158. [Crossref]

2. Komaroff AL (2017) Inflammation correlates with symptoms in chronic fatigue syndrome. Proc Natl Acad Sci U S A. 114(34): 8914-8916. [Crossref]

3. Poon DC, Ho YS, Chiu K, Wong HL, Chang RC (2015) Sickness: from the focus on cytokines, prostaglandins, and complement factors to the perspectives of neurons. Neurosci Biobehav Rev 57: 30-45. [Crossref]

4. VanElzakker MB (2013) Chronic fatigue syndrome from vagus nerve infection: a psychoneuroimmunological hypothesis. Med Hypotheses 81(3): 414-423. [Crossref]

5. Shoenfeld Y, Agmon-Levin N (2011) 'ASIA' - autoimmune/inflammatory syndrome induced by adjuvants. J Autoimmun 36: 4-8. [Crossref] 
6. Hotta O, Tanaka A, Torigoe A, Imai K, Ieiri N; Japanese Focal Inflammation Research Group (2017) Involvement of chronic epipharyngitis in autoimmune (autoinflammatory) syndrome induced by adjuvants (ASIA). Immunol Res 65: 66-71. [Crossref]

7. Horiguchi S (1975) The discovery of the nasopharyngitis and its influence on general diseases. Acta Otolaryngology 329(Supplement): 1-120.

8. Hotta $\mathrm{O}$, Inoue $\mathrm{CN}$, Tanaka A, Ieiri N (2017) The possible mechanism underling epipharyngeal abrasive therapy (EAT) with $\mathrm{ZnCl} 2$ solution for neuroendocrine disorders and autoimmune diseases. Journal of Antivirals \& Antiretrovirals 9(4): 81-86.

9. Sugita R (2010) A diagnostic method of the nasopharyngitis and a follow-up by cytology. Stomato-pharyngology 23(1): 23-35.

10. White SW, McRitchie RJ (1973) Nasopharyngeal reflexes: integrative analysis of evoked respiratory and cardiovascular effects. Aust J Exp Biol Med Sci 51: 17-31. [Crossref]

Copyright: (C2018 Hotta O. This is an open-access article distributed under the terms of the Creative Commons Attribution License, which permits unrestricted use, distribution, and reproduction in any medium, provided the original author and source are credited. 